\title{
Development and Implementation of a Clinical
} Pathway to Reduce Inappropriate Admissions Among Patients with Community-Acquired Pneumonia in a Private Health System in Brazil: An
Observational Cohort Study and a Promising Tool
for Efficiency Improvement

This article was published in the following Dove Press journal: Open Access Emergency Medicine

\author{
Rodrigo C Moreira $\mathbb{D}^{\prime}$ \\ Hugo T Mendonca-Filho ${ }^{2}$ \\ Ayla M Farias (iD) ${ }^{2}$ \\ Henry Sznejder ${ }^{3}$ \\ Eddy Lang ${ }^{4}$ \\ Margaret $M$ Wilson ${ }^{5}$ \\ 'Department of Clinical Research, United \\ Health Group, Rio de Janeiro, Brazil; \\ ${ }^{2}$ Clinical Intelligence, Amil Assistencia \\ Médica Internacional, Rio de Janeiro, Brazil; \\ ${ }^{3}$ Department of Analytics, Amil Assistencia \\ Médica Internacional, Rio de Janeiro, Brazil; \\ ${ }^{4}$ Emergency Medicine, University of \\ Calgary, Calgary, Canada; ${ }^{5}$ United \\ Healthcare Global, MO, USA
}

Purpose: Patients with community-acquired pneumonia (CAP) at low risk of death by CURB-65 scoring system are usually unnecessarily treated as inpatients generating additional economic and clinical burden. We aimed to implement an evidence-based clinical pathway to reduce hospital admissions of low-risk CAP and investigate factors related to mortality and readmissions within 30 days.

Patients and Methods: From November 2015 to August 2017, a clinical pathway was implemented at 20 hospitals. We included patients aged $>18$ years, with a diagnosis of CAP by the attendant physician. The main outcome was the monthly proportion of low-risk CURB-65 admission after the implementation of the clinical pathway. Logistic regression models were performed to assess variables associated with mortality and readmission in the admitted population within 30 days.

Results: We included 10,909 participants with suspected CAP. The proportion of low-risk CAP admitted decreased from $22.1 \%$ to $12.8 \%$ in the period. Among participants with low risk, there has been no perceptible increase in deaths $(0.80 \%)$ or readmissions $(6.92 \%)$. Regression analysis identified that CURB-65 variables, presence of pleural effusion $(\mathrm{OR}=1.74 ; 95 \% \mathrm{CI}=1.08-2.8$; $\mathrm{p}=0.02)$ and leucopenia $(\mathrm{OR}=2.47 ; 95 \% \mathrm{CI}=1.11-5.48 ; \mathrm{p}=0.02)$ were independently associated with 30-day mortality, whereas a prolonged hospital stay $(\mathrm{OR}=2.09 ; 95 \% \mathrm{CI}=1.14-3.83 ; \mathrm{p}=0.01)$ was associated with 30 -day readmission in the low-risk population.

Conclusion: The implementations of a clinical pathway diminished the proportion of lowrisk CAP admissions with no apparent increase in clinical outcomes within 30 days. Nonetheless, additional factors influence the clinical decision about the site of care management in low-risk CAP.

Keywords: community-acquired pneumonia, hospitalization, mortality, readmission

\section{Introduction}

Community-acquired pneumonia (CAP) is one of the most frequent diseases leading to hospital admissions. ${ }^{1}$ Despite being a worldwide-recognized cause of morbidity and a leading cause of death, it is frequently misdiagnosed or inappropriately 
managed. ${ }^{2,3}$ It is critical to improve our ability to rapidly diagnose and systematically integrate the use of prognostic aids to assess the severity of illness for those making treatment and disposition decisions. CURB-65 is a practical score validated to estimate mortality risk in pneumonia and, accordingly, stratifies patients into low risk who could be managed as outpatients in contrast to those at higher risk that would require admission to a greater level of intensive care. ${ }^{4}$

For a decade, despite guideline recommendations to increase the treatment of low-risk patients in the outpatient setting, as high as $44 \%$, of low-risk patients are still treated as inpatients. ${ }^{2,5}$ Social issues such as compliance with oral medical prescription and concern about a lack of follow-up care might influence physicians to decide against discharge as the initial approach. Notwithstanding, given its potential severity and expressive impact over healthcare costs it seems quite relevant to implement standards of care focused on selecting appropriate cases for hospitalization care or responsibly steerage to outpatient facilities and continuum care without impact on the morbimortality. ${ }^{6}$ For instance Blot S.I. et al showed that delays in oxygenation assessment negatively affect the time to antibiotic administration and consequently survival. ${ }^{7}$

Moreover, respiratory infections often occur as an epiphenomenon generating clinical instability in patients with chronic diseases such as heart failure or cancer. While it is fully justifiable to pay attention to these cases by assessing CAP severity along with clinical judgment, to understand inappropriate hospital admissions is key to implement future decisions to improve healthcare utilization. ${ }^{4,8,9}$

Given the CAP clinical relevance and impact over the healthcare chain providing an opportunity of costeffectiveness improvement, we aimed to understand CAP management in a real-world large population developing an evidence-based pathway, facing barriers in establish consensus and implementing a value-based policy focused on better clinical results. ${ }^{10}$ Hence, the objective of this study was specifically to investigate monthly trends of hospitalization in low-risk CAP patients after implementation of a clinical pathway, identifying factors associated with 30-day mortality in the admitted population and readmission among the low-risk patients. In addition, we explored factors related to mortality in specific subpopulations and assessed the performance of CURB-65 in patients with hypoxemia.

\section{Methods}

This was an observational cohort study with data collected retrospectively. Specifically, to address our main objective, which was to investigate the effect of the clinical pathway in monthly trends of hospitalization in low-risk CAP, we compared data before, that is, the first 3 months with the following months after the clinical pathway implementation. United Health Group (UHG) operates a large health care network in Brazil, including 33 owned hospitals and 95 medical centers or outpatient facilities. These delivery centers are core to the business and a big effort has been applied to encourage evidence-based care to patients. All clinical and data claim flow from this healthcare system and have been converged to an organized single layer, a patient-centric database called Clinical Data Warehouse (CDW).

\section{Clinical Pathway Development and Implementation Process}

The clinical pathway development started with an evidencebased group of health care professionals who started working defining and structuring several major questions about CAP according to a standardized methodology. ${ }^{11}$ A literature search included English and Portuguese references. ${ }^{12}$ Quality of evidence was assessed using the GRADE system, which ranks an article based, for example, on the risk of bias, variability in results from different studies and indirectness of evidence. ${ }^{13}$ Then, a draft of the Clinical Pathway in CAP was constructed and compared with major guidelines ${ }^{2,14,15}$ tracking possible and predictable barriers for implementation in our hospitals. The CAP pathway draft and related references were sent to the participants 2 weeks prior to a consensus meeting to allow their appraisal and to stimulate additional questions to arise. The consensus-meeting day included $70 \%$ of the most relevant healthcare professionals and local stakeholders, invited based on their relevance for clinical assistance, confidentiality agreement, compliance with evidence-based clinical practice and commitment with effective implementation. To generate the consensus, we considered the strength of the evidence supporting each structured question about CAP management, using the Delphi modified method to arrive at a decision. Briefly, after the clinical pathway draft overview, we offered objective questions together with the supporting evidence to participants for discussion in a plenary interactive session, until they came up with a decision. A result was consolidated for each question, assuming $65 \%$ of agreement as a trigger for consensus. 
At the end of the meeting day, the final clinical pathway was assumed as agreed and ready for implementation. ${ }^{16}$ The clinical pathway enclosed the following orientations (i) once a patient presented at the emergency department with a combination of signs and symptoms suggestive of PAC such as cough, chest pain, myalgia, fever, tachypnea, rales, chills, sweating, headache or confusion it was recommended to measure the peripheral oxygen saturation and take a chest $\mathrm{X}$-ray. At this point, it was necessary (ii) to evaluate the severity of disease assessed using the CRB-65/CURB-65 score and the indication of intravenous venous antibiotic or volume replacement as needed. If the patient was considered for admission it was necessary to take a hemogram, serum biochemistry, electrolyte profile, arterial blood gas test and hemoculture. For patients assigned as low risk, it was advised to treat them as outpatient with a reevaluation scheduled. Regarding patients with moderate risk, the consideration was a short stay in the emergency room and to treat them as outpatient or admission depending on the general practitioner's initial assessment. For high-risk subjects, besides hospital admission, it was required to start antibiotics within 1 hour and (iii) a careful evaluation for intensive care unit (ICU) treatment if signs of hemodynamic or ventilatory instability were present such as respiratory rate $\geq 30 \mathrm{bpm}$, hypoxemia or hypotension requiring volume replacement. Likewise, were indicators of ICU the existence of pulmonary infiltrates with pleural effusion or mental confusion; urea $>50 \mathrm{mg} / \mathrm{dL}$; low platelet or leukocyte count; hypothermia, hyperlactatemia or acidosis. A comprehensible schematic representation of the development of CAP pathway is shown in supplementary Figure S1.

Doctors were not obligated to follow the clinical pathway. However, a start-up session allowed hospitals and leadership staff to discuss and became familiar with the pathway. Then, case managers nurses started continuous monitoring of clinical variables and outcomes, making the representatives physicians know about each non-conform case. Every other week, all cases were presented in a remote meeting by case managers and reviewed under the supervision of an engagement supervisor. A face-to-face bimonthly debriefing meeting allowed a deep dive in each hospital performance aiming to achieve a higher level of alignment.

\section{Participant's Enrolment}

The clinical pathway was implemented at 20 UHG hospitals at different moments, from November 2015 to August 2017 and the last follow up terminated in January 2018. We included all individuals who presented to the emergency room (ER) with suspected CAP within 20 UHG owned hospitals committed to CAP Clinical Pathway.

To be enrolled in the study, the patient had to be aged higher than 18 years, with a diagnosis of suspected CAP made by the attendant physician, specified by the International Classification of Diseases (ICD-10). The attendant physician made decisions concerning the initial approach such as inpatient, outpatient treatment or transfer from ER.

\section{Data Collection and Covariates Definitions}

Trained research nurses collected clinical data from medical charts, which included patient age, initial vital signs and mental status, comorbid conditions, pertinent laboratory test results, and chest radiography findings from ED presentation. To aid nurse's work and avoid data entry errors all these variables except for age, were collected dichotomized in a standardized sheet and then at the data-coordinating center processed into a database for quality control evaluation where they were checked for validity and consistency. Additional information such as date of birth, sex, vital status, discharge diagnosis and readmission within 30 days after discharge were extracted from CDW health records.

Variables were defined or calculated as follows. For the diagnosis of suspected CAP, we used health records information containing ICD-10 codes J15 and J18; for Influenza and pneumonia, we used ICD-10 of discharge comprising J09.X1, $\mathrm{J} 10$, and $\mathrm{J} 11$ codes and confirmed pneumonia was restricted to those participants presenting with symptoms of CAP and a new infiltrate found on chest radiography. Age was dichotomized into 65 years or older, gender female vs male, leucocytosis or leucopenia when the white blood cell count was higher 12,000 cell $/ \mathrm{mm} 3$ or less than $<4000$ cell $/ \mathrm{mm} 3$, respectively, hypotension if systolic blood pressure $<90 \mathrm{~mm} \mathrm{Hg}$ or diastolic $\leq 60 \mathrm{mmHg}$; hypoxemia at admission was defined as present when the oxygen saturation measured by pulse oximetry (SpO2) was less then $90 \%$ and hypothermia when body temperature less than $36^{\circ}$. Thrombocytopenia was determined when the platelet count was below $100.000 / \mathrm{mm}^{3}$. Hypotension requiring fluid resuscitation was defined when it was prescribed intravenous fluids for patients with hypotension. A prolonged hospital stay was ascertained when the length of stay was more than six days.

Severity of CAP at admission was calculated according to CURB-65 criteria (confusion, urea $>50 \mathrm{mmol} / \mathrm{l}$, respiratory rate $>30 / \mathrm{min}$, and hypotension: diastolic blood pressure (DBP) $<60 \mathrm{~mm} \mathrm{Hg}$ or systolic blood pressure (SBP) 
$<90 \mathrm{~mm} \mathrm{Hg}$ ). In cases where patients were treated as outpatients and not admitted to hospitals, complementary exams, which include a blood sample, were not routinely collected bringing limited availability for urea, leukogram and platelet count results. In such cases, the CRB-65 was used. We categorized the severity of pneumonia into three classes of CRB-65/CURB-65 score: low risk, 0-1; moderate, 2 ; high risk, $\geq 3$.

\section{Outcomes}

The primary outcome was the monthly proportion of lowrisk CRB-65/CURB-65 admission into hospitals post implementation of the clinical pathway. The secondary outcome was 30-day adjusted all-cause mortality and readmission in the hospitalized population. The exploratory analysis included the ascertainment of CURB-65 prognostic performance in participants admitted and non-admitted with hypoxemia.

\section{Statistical Analysis}

To analyze the variation of the proportion of low-risk patients with CAP admitted to the hospitals we plotted a monthly time trend with the estimated regression line. Descriptive statistics of the population included the median and interquartile range for continuous variables and frequency distributions for categorical variables. We created a table to compare the characteristics between categories of CURB-65 and alive and dead patients in the cohort at the end of follow-up. Differences were assessed with the nonparametric Mann-Whitney test to compare continuous variables and Chi-squared tests for categorical variables with the corresponding p-values. Logistic regression models with the calculated odds ratio (OR) and $95 \% \mathrm{CI}$ were performed for multivariate analysis in the subgroup of participants who were admitted to the hospitals to determine which variables were associated with mortality within 30 days. Patients with missing data were excluded from the regression models. From the explanatory variables, a generalized linear model function built all possible unique models involving these variables and the best fit model explaining 30-day mortality in admitted patients with CAP, was chosen using an automated model selection based in a genetic algorithm ranked by the Akaike information criterion. ${ }^{17}$ The same procedure was carried out to identify factors independently related to readmission among the admitted low-risk CAP subjects, mortality in those admitted with confirmed pneumonia and mortality in individuals with low risk that were admitted to the hospitals. To compare the accuracy of CURB-65 tool in predicting death according to the presence of hypoxemia we fit a logistic regression model and incorporated the predicted values into the dataset. Afterward, to assess model discrimination, we constructed a receiver operating characteristic (ROC) curve calculating the area under the curve (AUC) for the entire sample and segregated for subjects with and without hypoxemia. The optimal cut-off point was determined by "youden" index. Furthermore, for each of the five points of CURB-65, we computed the sensitivities and specificities with the respective confidence intervals. ${ }^{18}$ This study was conducted according to the Declaration of Helsinki principles and the study was approved by the Comitê de Ética em Pesquisa do Hospital Pró-Cardíaco, the local institutional review board (Reference number 890). Data were anonymised for the analysis and all tests were 2-tailed assuming significance if $\mathrm{p}<0.05$, performed in the $\mathrm{R}$ environment version 3.2.2.

\section{Results}

We included 10,909 participants who attended on ED suspected of CAP from our owned network and follow up was considered complete in $99.95 \%$ of patients for mortality and $90.96 \%$ for readmission. Looking into CDW database, we found 19 cases of influenza in the period, and among 6290 participants whose ICD-10 discharge code was available, we found a considerable inconsistency in the final diagnosis. Nevertheless, the most frequent diagnosis was pneumonia (J15 and J18) with $1804(28.67 \%)$ cases and the related diagnosis of sepsis (A41) with 339 (5.38\%) cases but we also identified events of angina pectoris (I20) and heart failure (I50) with 249 (3.95\%) and 163 (2.95\%) occurrences, respectively.

\section{Trends in Admission of Patients with Low-Risk Suspected CAP}

The mean follow-up time of observation was 11.3 months. In summary, among each of the 20 hospitals, eight demonstrated some extent of reduction in the admission of patients with CAP and low-risk CURB-65 score over time, two revealed an increase in the proportion of admissions of those patients and nine remained stable after implementation of the clinical pathway. Taken together these results indicates that the monthly proportion of patients with low-risk CAP that were admitted to the hospitals decreased significantly over time after implementation of the clinical pathway, ranging from 350 patients out of 1583 (22.15\%) in the first 3 months post implementation of the clinical pathway to 
40 out of $308(12.99 \%)$ when we assume the cumulative period of the twenty-first month to the end of the study $(p=0.04)$. The monthly trend with the respective regression line for the proportion of patients with low risk admitted to the hospitals is depicted in Figure 1 [For more details, also see supplementary Table S1]. Among 9003 participants with a CURB-65 score of 0 and 1, there has been no perceptible increase in clinical outcomes since only $72(0.80 \%)$ died and $568(6.92 \%)$ were readmitted within 30 days. The proportion was even lower among those who were treated as outpatients $(0.12 \%$ and $5.6 \%$ for death and readmission within 30 days, respectively). The clinical and laboratory characteristics of patients at the end of follow up are depicted in Table 1

\section{Overall Population Characteristics}

The population was predominantly female (57.79\%) with a median age of 59 years [Interquartile range (IQR) $40-76]$, most of them less than 65 years old $(60.07 \%)$ with a small proportion showing COPD as comorbidity (7.25\%). The most common signs and symptoms during patient evaluation on ED were cough $(87.98 \%)$, expectoration or dyspnea $(69.27 \%)$, pulmonary rales $(61.39 \%)$ and fever $(42.98 \%)$. A new focal infiltrate suggestive of PAC on chest radiography was found in almost half of participants $(49.42 \%)$ and signs of disease severity such as multilobar or diffuse pattern and pleural effusion were present in $12.47 \%$ and $6.1 \%$ of roentgenograms, respectively.

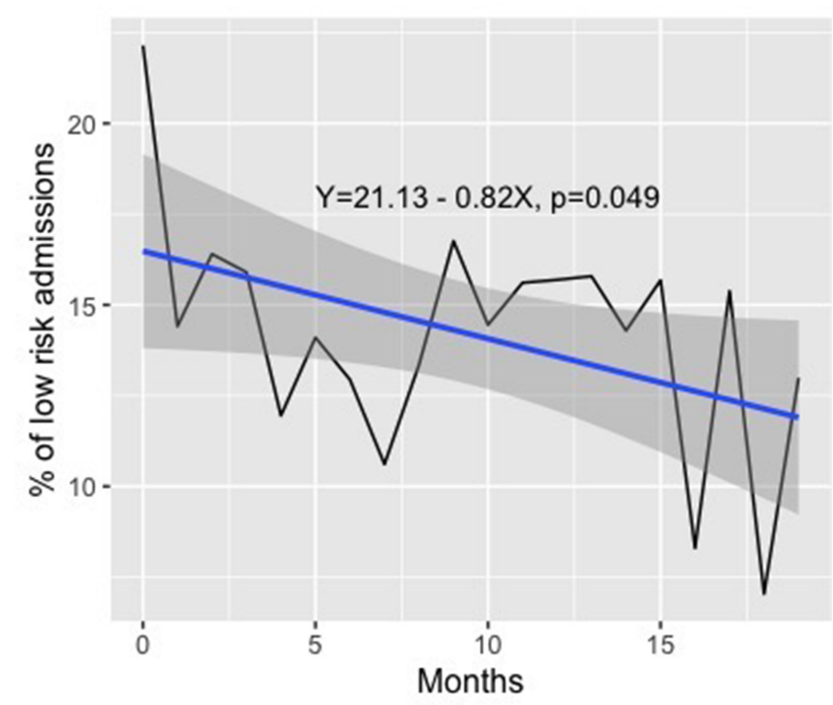

Figure I Monthly trends with the respective regression line for the proportion of patients with low risk admitted to the hospitals from November 2015 to January 2018* Notes: *The denominator for the proportion is the total number of patients assigned as low-risk CURB-65 in the emergency department. The regression line was derived from a linear model.
Although the greater majority of subjects were eligible to be managed as outpatients according to CRB-65/CURB-65 the observed proportion depending on the physician decision was $84.62 \%$. By contrast, a small proportion of patients initially exhibited a combination of respiratory rate $>30$ breaths/min $(2.83 \%)$, leukopenia resulting from infection $(2.91 \%)$ or hypotension requiring fluid resuscitation $(3.20 \%)$ which would eventually determine their admission to ICU $(9.88 \%)$. It is interesting to notice that regarding participants with low-risk CRB-65/CURB-65 although most of them had been treated as outpatients there was a small fraction (4.62\%) requiring ICU stay. At the end of 30 days, 267 (2.45\%) patients of the overall population were dead and $845(8.52 \%)$ were readmitted at the hospital yielding a LOS equal or more than 6 days of $14.30 \%$, for patients with low risk the mortality was considered minimal $(0.8 \%)$ (Table 1$)$. When we take into account only the 2750 participants who were admitted, $1385(50.36 \%)$ were considered low risk and 416 (15.12\%) admitted to ICU; among those admitted with low risk $63(4.56 \%)$ died and 177 (14.39\%) were readmitted within 30 days.

\section{Determinants of Mortality in Patients Admitted with CAP}

Confirmed CAP was present in $74.02 \%$ of the population admitted to the hospitals. In crude analysis, patients who were dead within 30 days were predominantly older than 65 years when compared to patients who were alive $(83.92 \%$ vs $63 \% ; \mathrm{p}<0.001)$. They also had more clinical signs of CAP including dyspnea $(85.6 \%$ vs $80.56 \% ; \mathrm{p}<0.001)$, rales $(88.49 \%$ vs $80.73 \% ; \mathrm{p}<0.001)$ and confusion $(33.06 \%$ vs $14.37 \% ; \mathrm{p}<0.01)$. In the chest $\mathrm{X}$-ray evaluation, pleural effusion was also more frequently found in subjects who died when compared to those who were alive (25.98\% vs $16.36 \%$, $\mathrm{p}<0.001)$. Disease severity was also marked prevalent in individuals who died, which comprised urea $>50 \mathrm{mg} / \mathrm{dl}$ $(63.38 \%$ vs $42.02 \%$; $<0.001)$, respiratory frequency higher than 30 breaths per $\min (21.03 \%$ vs $9.41 \%, \mathrm{p}<0.001)$ and hypotension (33.46\% vs $20.33 \%$; $<<0.001$ ); consequently, the proportion of participants with CURB-65 categorized as high risk was more frequent in these patients $(44.71 \%$ vs $17.15 \% ; \mathrm{p}<0.001)$ as well as ICU admission (63.92\% vs $36.67 \%$; $<0.001$ ). (Table 2)

The final multivariate logistic regression analysis identified that CURB-65 variables were independently associated with 30 -day mortality in the admitted population 
Table I Comparison of Demographic Characteristics Among Subjects with Suspect CAP by CRB-65 Risk Score

\begin{tabular}{|c|c|c|c|c|c|c|}
\hline & Low & Moderate & High & Total & $\mathbf{P}_{\text {value }}{ }^{\mathbf{a}}$ & Missing \\
\hline Total & 9003 & 1305 & 601 & 10,909 & & \\
\hline Age median (IQR) & $53(37.69)$ & $80(72.86)$ & $83(77.89)$ & $59(40.76)$ & $<0.001$ & $440(4.03)$ \\
\hline Sex: Female & $5146(58.62)$ & 689 (54.77) & $302(5 \mid .7 I)$ & $6137(57.79)$ & $<0.001$ & $289(2.64)$ \\
\hline Age $>65$ years & $2566(28.5 I)$ & $1214(93.1)$ & 585 (97.34) & $4365(40.03)$ & $<0.001$ & $440(4.03)$ \\
\hline COPD & $426(5.13)$ & $189(15.13)$ & $120(20.58)$ & $735(7.25)$ & $<0.001$ & $777(7.12)$ \\
\hline Cough & 7955 (89.2) & $1084(84.23)$ & 457 (77.72) & $9496(87.98)$ & $<0.001$ & $116(1.06)$ \\
\hline Expectoration or dyspnea & $5940(67.19)$ & $997(77.35)$ & $490(82.77)$ & 7427 (69.27) & $<0.001$ & $117(1.07)$ \\
\hline Chest pain & $2744(31.63)$ & $247(20.11)$ & $102(18.15)$ & $3093(29.56)$ & $<0.001$ & $445(4.07)$ \\
\hline Rales & $5082(57.4)$ & 999 (77.56) & $510(85.71)$ & $6591(61.39)$ & $<0.001$ & $172(1.57)$ \\
\hline Confusion & $23(0.26)$ & 171 (13.39) & $301(50.42)$ & $495(4.6)$ & $<0.001$ & $155(1.42)$ \\
\hline Headache & $1664(20.72)$ & $91(8.38)$ & $34(6.92)$ & $1789(18.62)$ & $<0.001$ & $1303(11.94)$ \\
\hline Sweating & $244(3.15)$ & $25(2.36)$ & $25(5.08)$ & $294(3.16)$ & 0.0172 & 1614 (14.79) \\
\hline Chills & $548(7.03)$ & $88(8.22)$ & $51(10.24)$ & $687(7.34)$ & 0.0142 & $1545(14.16)$ \\
\hline Myalgia & $2165(26.11)$ & $216(19.01)$ & $85(|6.3|)$ & $2466(24.78)$ & $<0.001$ & 959 (8.79) \\
\hline Fever $^{\mathrm{b}}$ & $3858(43.77)$ & 477 (37.77) & $248(42.47)$ & $4583(42.98)$ & $<0.001$ & $247(2.26)$ \\
\hline New pulmonary infiltrates & $3358(45.7 I)$ & $698(61.88)$ & $395(74.11)$ & 4451 (49.42) & $<0.001$ & $1902(17.43)$ \\
\hline Multilobar infiltrates & $703(9.88)$ & $231(20.92)$ & $155(30.1)$ & $1089(12.47)$ & $<0.001$ & $2174(19.92)$ \\
\hline Pleural effusion & $318(4.47)$ & $145(13.17)$ & $69(13.58)$ & $532(6.1)$ & $<0.001$ & $2183(20.01)$ \\
\hline Respiratory rate $>30$ breaths $/ \mathrm{min}$ & $54(0.61)$ & $86(6.65)$ & $164(27.38)$ & $304(2.83)$ & $<0.001$ & $163(1.49)$ \\
\hline Hypotension ${ }^{c}$ & $264(2.98)$ & $357(27.53)$ & $388(64.77)$ & $1009(9.37)$ & $<0.001$ & $145(1.32)$ \\
\hline Hypotension requiring fluid resuscitation & $78(0.9)$ & $88(6.89)$ & $170(28.76)$ & $336(3.2)$ & $<0.001$ & $414(3.79)$ \\
\hline Hypothermia $^{d}$ & $288(3.29)$ & $79(6.26)$ & $58(9.86)$ & $425(4.01)$ & $<0.001$ & $301(2.75)$ \\
\hline Assignment & & & & & $<0.001$ & 0 \\
\hline Outpatient & $7618(84.62)$ & $483(37.01)$ & $58(9.65)$ & 8159 (74.79) & & \\
\hline General ward & $935(10.39)$ & $483(37.01)$ & $184(30.62)$ & $1602(14.69)$ & & \\
\hline Transferred & $34(0.38)$ & $24(1.84)$ & $12(2)$ & $70(0.64)$ & & \\
\hline ICU & $416(4.62)$ & $315(24.14)$ & $347(57.74)$ & $1078(9.88)$ & & \\
\hline Antibiotic therapy within $60 \mathrm{~min}$ & $1207(13.57)$ & $541(42.13)$ & $424(72.35)$ & $2172(20.18)$ & $<0.001$ & $147(1.34)$ \\
\hline Hemoculture & $881(10.23)$ & $518(41.01)$ & $403(69.13)$ & $1802(17.23)$ & $<0.001$ & $450(4.12)$ \\
\hline 30-day mortality & $72(0.8)$ & $80(6.13)$ & $115(19.2)$ & $267(2.45)$ & $<0.001$ & $5(0.04)$ \\
\hline 30-day readmission & $568(6.92)$ & $180(15.36)$ & $97(18.06)$ & $845(8.52)$ & $<0.001$ & $986(9.03)$ \\
\hline LOS $>6$ days & 709 (7.89) & $501(38.75)$ & $345(58.47)$ & $1555(14.3)$ & $<0.001$ & $37(0.33)$ \\
\hline
\end{tabular}

Notes: Columns provide number (percentage) of each group unless otherwise specified. Severity of pneumonia was assessed using CRB-65/CURB-65 score divided into low risk, $0-1$; moderate, 2 and high risk, $\geq 3$. ${ }^{\mathrm{a}}$ Chi-squared for categorical variables and Mann-Whitney tests for continuous variables. ${ }^{\mathrm{b}} \mathrm{Body}$ temperature $>38^{\circ} \mathrm{C}$. ${ }^{\mathrm{C} S y s t o l i c}$ blood pressure $<90 \mathrm{~mm} \mathrm{Hg}$; or diastolic $\leq 60 \mathrm{mmHg}$. ${ }^{d}$ Body temperature $<36^{\circ} \mathrm{C}$.

Abbreviations: COPD, chronic obstructive pulmonary disease; ICU, intensive care unit; LOS, length of stay.

with the exception of hypotension. In addition, the presence of pleural effusion $(\mathrm{OR}=1.74 ; 95 \% \mathrm{CI}=1.08-2.8$; $\mathrm{p}=0.02)$ and leucopenia $(\mathrm{OR}=2.47 ; 95 \% \mathrm{CI}=1.11-5.48$; $\mathrm{p}=0.02$ ) were predictive of 30 -day mortality in patients admitted with suspected CAP (Table 3). Among participants admitted with low-risk CURB-65, the predictors of mortality within 30 days were new pulmonary infiltrates $(\mathrm{OR}=3.1 ; 95 \% \mathrm{CI}=1.08-8.89 ; \mathrm{p}=0.03)$, leucopenia $(\mathrm{OR}=$ $6.37 ; 95 \% \mathrm{CI}=2.14-18.94 ; \mathrm{p}<0.01)$, and a prolonged hospital stay $(\mathrm{OR}=5.4 ; 95 \% \mathrm{CI}=2.17,13.45 ; \mathrm{p}<0.01)$ [For more detail, see Supplementary Table S2]. Likewise, for patients admitted with confirmed pneumonia ( $\mathrm{n}=1795)$, apart from CURB-65 variables, pleural effusion $(\mathrm{OR}=1.76 ; 95 \% \mathrm{CI}=1.01-3.01 ; \mathrm{p}=0.04)$, thrombocytopenia $(\mathrm{OR}=2.78 ; 95 \% \mathrm{CI}=0.94-7.19 ; \mathrm{p}=0.04)$ and ICU stay
$(\mathrm{OR}=2.44 ; 95 \% \mathrm{CI}=1.43-4.23 ; \mathrm{p}=<0.01)$ were associated with increased mortality within 30 days [For more detail, see supplementary Table S3].

\section{Predictors of 30-Day Readmission in Patients with Low-Risk CAP}

In Adjusted analysis, among patients that were admitted with low-risk CAP, a prolonged hospital stay of more than 6 days was independently associated with an increased chance of 30-day readmission $(\mathrm{OR}=1.58 ; 95 \% \mathrm{CI}=1.03-2.41 ; \mathrm{p}=0.03)$, albeit cough had a protective effect $(\mathrm{OR}=0.53 ; 95 \%$ $\mathrm{CI}=0.32-0.89 ; \mathrm{p}=0.02$ ). The clinical and laboratory variables associated with hospital readmission among the low risk admitted CAP patients are shown in Table 4. 
Table 2 Comparison of Clinical and Laboratory Characteristics Among Participants with Suspected CAP That Were Admitted to the Hospitals According to 30-Day Mortality

\begin{tabular}{|c|c|c|c|c|}
\hline & Alive & Dead & Total & P value ${ }^{a}$ \\
\hline Total & 2490 & 255 & 2750 & \\
\hline Age median (IQR) & $74(56.84)$ & $80.5(70.87)$ & $75(58.85)$ & $<0.001$ \\
\hline Sex: Female & I $320(54.68)$ & $125(51.44)$ & I445 (54.38) & 0.3337 \\
\hline Age $\geq 65$ years & $1568(63)$ & $214(83.92)$ & I782 (64.94) & $<0.001$ \\
\hline COPD & $394(16.55)$ & $49(20.33)$ & $443(16.9)$ & 0.136 \\
\hline Cough & $2009(82.37)$ & $162(65.59)$ & $2|7|(80.83)$ & $<0.001$ \\
\hline Expectoration or dyspnea & $1956(80.56)$ & $214(85.6)$ & $2170(81.03)$ & 0.0529 \\
\hline Rales & I $977(80.73)$ & $223(88.49)$ & $2200(81.45)$ & 0.0025 \\
\hline Fever $^{\beta}$ & II 79 (49.02) & $84(34.7 I)$ & I263 (47.7I) & $<0.001$ \\
\hline Chest pain & $610(26.12)$ & $33(14.47)$ & $643(25.09)$ & $<0.001$ \\
\hline Confusion & $352(14.37)$ & $82(33.06)$ & $434(16.09)$ & $<0.001$ \\
\hline Headache & $206(9.92)$ & $9(4.33)$ & $215(9.4 I)$ & 0.008 \\
\hline Sweating & $87(4.28)$ & $7(3.33)$ & $94(4.19)$ & 0.5156 \\
\hline Chills & $221(10.68)$ & $6(2.88)$ & $227(9.97)$ & $<0.001$ \\
\hline Myalgia & $45 I(20.56)$ & $18(8.37)$ & $469(19.47)$ & $<0.001$ \\
\hline New pulmonary infiltrates & I633 (73.89) & $159(75.36)$ & I792 (74.02) & 0.6431 \\
\hline Multilobar infiltrates & $567(26.82)$ & $64(31.22)$ & $631(27.21)$ & 0.1767 \\
\hline Pleural effusion & $34 I(16.36)$ & $53(25.98)$ & $394(17.22)$ & $<0.001$ \\
\hline Leucocitosis $^{b}$ & $1030(43.5)$ & $112(47.06)$ & II 42 (43.82) & 0.2911 \\
\hline Leucopenia $^{c}$ & $89(3.76)$ & $13(5.46)$ & $102(3.92)$ & 0.1969 \\
\hline Urea $>50 \mathrm{mg} / \mathrm{dl}$ & $943(42.02)$ & $157(67.38)$ & I I $00(44.4$ I) & $<0.001$ \\
\hline Respiratory rate $>30$ breaths $/ \mathrm{min}$ & $23 I(9.4 I)$ & $53(21.03)$ & $284(10.49)$ & $<0.001$ \\
\hline Hypotension $^{d}$ & $500(20.33)$ & $85(33.46)$ & $585(21.55)$ & $<0.001$ \\
\hline CURB-65 & & & & $<0.001$ \\
\hline Low & $1319(52.97)$ & $63(24.7 I)$ & 1382 (50.35) & \\
\hline Moderate & $744(29.88)$ & 78 (30.59) & $822(29.95)$ & \\
\hline High & $427(17.15)$ & II 4 (44.7I) & $54 I(19.7 I)$ & \\
\hline Hypothermia ${ }^{\mathrm{e}}$ & $136(5.66)$ & $26(10.79)$ & $162(6.13)$ & 0.0016 \\
\hline Thrombocytopenia $^{f}$ & $98(4.21)$ & $18(7.63)$ & $116(4.52)$ & 0.0159 \\
\hline Hypotension requiring fluid resuscitation & $232(9.59)$ & $55(22.27)$ & $287(10.77)$ & $<0.001$ \\
\hline Assignment & & & & $<0.001$ \\
\hline General ward & I507 (60.52) & $92(36.08)$ & $1599(58.25)$ & \\
\hline Transferred & $70(2.8 I)$ & $0(0)$ & $70(2.55)$ & \\
\hline ICU & $913(36.67)$ & $163(63.92)$ & $1076(39.2)$ & \\
\hline Antibiotic therapy within $60 \mathrm{~min}$ & I 446 (59.9) & $179(7 \mid .6)$ & $1625(61)$ & $<0.001$ \\
\hline Hemoculture & $1402(59.58)$ & $164(67.49)$ & I566 (60.32) & 0.0165 \\
\hline LOS $>6$ days & $1384(56.31)$ & $169(66.27)$ & I553 (57.24) & 0.0022 \\
\hline
\end{tabular}

Notes: Columns provide number (percentage) of each group unless otherwise specified. ${ }^{\mathrm{a}}$ Chi-squared for categorical variables and Mann-Whitney tests for continuous variables. ${ }^{\beta}$ Body temperature $\geq 38.0^{\circ} \mathrm{C}$. ${ }^{b}$ White blood cell count $>12,000 \mathrm{cell} / \mathrm{mm}^{3}$. ${ }^{C}$ White blood cell count $<4000 \mathrm{cell} / \mathrm{mm}{ }^{3}$. ${ }^{d}$ Systolic blood pressure $<90 \mathrm{~mm} \mathrm{Hg}$ or diastolic $\leq 60 \mathrm{mmHg}$. ${ }^{e}$ Body temperature $<36^{\circ} \mathrm{C}$. 'Platelet count below $100,000 \mathrm{~mm}^{3}$.

Abbreviations: COPD, chronic obstructive pulmonary disease; ICU, intensive care unit; LOS, length of stay.

\section{Accuracy of CURB-65 Tool in the Presence of Hypoxemia}

Among 3425 participants in which data about oxygen saturation were available, those presenting with hypoxemia, showed significantly reduced accuracy $(\mathrm{AUC}=72.68$, $95 \% \mathrm{CI}=65.54-79.83)$ when compared to patients without hypoxemia [(AUC=86.42, 95\% CI $=82.97-89.88)$; $\mathrm{p}<0.01]$. For a threshold $\geq 2$, which identifies patients with intermediate and high risk of mortality, the score demonstrated similar sensitivity (0.97 vs 0.97$)$ and lower specificity ( 0.45 vs 0.78$)$ for identifying patients who died within 30 days. An additional table and figure files show this in more details [See supplementary Table S4 and supplementary Figure S2, respectively]. 
Table 3 Final Logistic Regression Model for the Prediction of 30Day Mortality in Patients Admitted with Suspected CAP

\begin{tabular}{|c|c|c|c|}
\hline Variables & OR & $95 \% \mathrm{Cl}$ & P value \\
\hline Sex: Male & 1.45 & $0.98-2.17$ & 0.0659 \\
\hline Age $\geq 65$ years & 2.49 & $1.39-4.45$ & 0.002 \\
\hline Cough & 0.52 & $0.34-0.81$ & 0.0034 \\
\hline Chest pain & 0.65 & $0.38, I . I I$ & 0.1205 \\
\hline Rales & 1.43 & $0.77-2.66$ & 0.2570 \\
\hline Confusion & 1.86 & I. $17-2.97$ & 0.0089 \\
\hline Chills & 0.33 & $0.12-0.94$ & 0.0384 \\
\hline Fever & 0.62 & $0.4-0.96$ & 0.0315 \\
\hline Muiltilobar & 1.48 & $0.97-2.26$ & 0.0687 \\
\hline \multicolumn{4}{|l|}{ Infiltrates } \\
\hline Pleural effusion & $\mathrm{I} .74$ & $1.08-2.80$ & 0.0227 \\
\hline Leucopenia $^{\mathrm{a}}$ & 2.47 & $\mathrm{I} . \mathrm{II}-5.48$ & 0.0260 \\
\hline Urea $>50 \mathrm{mg} / \mathrm{dl}$ & 1.72 & $1.11-2.66$ & 0.0144 \\
\hline Respiratory rate $>30$ breaths $/ \mathrm{min}$ & 1.88 & $1.13-3.13$ & 0.0147 \\
\hline Hypotension ${ }^{\mathrm{b}}$ & 1.50 & $0.98-2.3$ & 0.0627 \\
\hline Thrombocytopenia $^{c}$ & 1.93 & $0.8-4.65$ & 0.1447 \\
\hline Assignment: UCl & 2.18 & $1.38-3.44$ & $<0.001$ \\
\hline LOS & 0.89 & $0.58-1.38$ & 0.6117 \\
\hline
\end{tabular}

Notes: ${ }^{\text {aW }}$ hite blood cell count $<4000$ cell $/ \mathrm{mm}^{3}$. bystolic blood pressure $<90 \mathrm{~mm}$

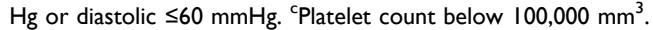

Abbreviations: ICU, intensive care unit; LOS, length of stay.

\section{Discussion}

In this prospective real-world study, we demonstrated that the proportion of low-risk CAP admissions decreased significantly over time after the implementation of the clinical pathway. Moreover, among participants with low-risk CURB-65, there has been no apparent increase in clinical outcomes within 30 days. We also found additional predictors of mortality in patients with suspected CAP aside from CURB-65 variables comprising low leucocyte cell count and chest radiography abnormalities. For the 30-day risk of readmission, a prolonged hospital stay was the main predictor. Furthermore, CURB-65 showed reduced performance in patients with hypoxemia.

To our knowledge, this is the first Brazilian experience in implementing a targeted CAP Clinical Pathway in order to optimize health system performance, designed and conducted by a health organization in cooperation with an expressive portion of healthcare provider network. Regarding severity stratification scores, CURB 65 was considered more suitable and chosen especially because of its presumably higher adherence in clinical practice, since the PSI is significantly more complex, requiring the measurement of 20 parameters. Evidence suggests that clinicians find it difficult to use and it is currently underutilized. ${ }^{19}$ The IDSA/ATS and British Thoracic Society (BTS) guidelines
Table 4 Clinical and Laboratory Variables Associated with Hospital Readmission Among the Low-Risk Admitted CAP Patients

\begin{tabular}{|l|l|l|l|}
\hline Variables & OR & $\mathbf{9 5 \%} \mathbf{~ C I}$ & P value \\
\hline Sex: Female & 1.25 & $0.82-1.92$ & 0.2897 \\
Cough & 0.53 & $0.32-0.89$ & 0.0158 \\
Rales & 1.21 & $0.71-2.07$ & 0.4776 \\
New pulmonary & 0.92 & $0.58-1.46$ & 0.7232 \\
Infiltrates & & & \\
Pleural effusion & 1.02 & $0.6-1.74$ & 0.9426 \\
COPD & 1.51 & $0.84-2.73$ & 0.1695 \\
Age $\geq 65$ years & 1.06 & $0.68-1.64$ & 0.8104 \\
Urea $>50 \mathrm{mg} / \mathrm{dl}$ & 1.24 & $0.63-2.48$ & 0.5330 \\
LOS $>6$ days & 1.58 & $1.03-2.41$ & 0.0352 \\
\hline
\end{tabular}

Abbreviations: COPD, chronic obstructive pulmonary disease; LOS, length of stay.

recommend that patients with scores of less than or equal to one are at low risk of clinical complications and seemingly qualified to be managed as outpatients. ${ }^{2,20}$ Despite that, according to baseline data of our population, more than $20 \%$ of CURB 65 categorized as $0-1$ used to be inappropriately admitted to the hospital. Other series of cases reported that as high as $61 \%$ of the low-risk CURB-65 was admitted. ${ }^{21}$ In the present experience, it became clear that continuous educational initiatives covering stakeholders and institutional support improved clinical engagement changing behavior regarding avoidable admissions with an absolute reduction of $9.1 \%$ in admission rate among the low-risk population at the end of study period.

The most frequently mentioned reasons to admit low-risk patients included unreliable access to the prescribed antimicrobials or continuity of care, particularly for the low-income population. Despite lacking a pharmacy benefit management program, careful selection and patient referral to outpatient facility network might have had a positive neutralizing effect on death and 30-day readmission registered as $0.12 \%$ and $5.6 \%$ among patients with low-risk CAP, respectively. These results are in agreement with the original cohort from Lim et al, who reported a low mortality rate of $1.5 \%{ }^{9}$

Inaccurate diagnosis is a well-recognized issue in CAP patients approach at the ER and it is known that IDSA clinical criterion lacks specificity. In the present study, new pulmonary infiltrates were noted only in $49.41 \%$ of CAP cases. These numbers are in line with a primary-care-based study by Blaeuer et $\mathrm{al}^{22}$ but slightly differs from the results presented by Vugt et al, who found radiographic pneumonia in $57 \%$ of patients with clinically suspected pneumonia. ${ }^{23}$ In all of these studies, the diagnosis of pneumonia was based on 
clinical suspicion by the attending physician, based on lower respiratory symptoms, reflecting a real-world scenario.

Although the overall mortality and readmission within 30 days were low, among the admitted participants an unexpected proportion of low-risk CAP presented with other markers of severity of the disease requiring intensive care and ICU admission (15.12\%). In our population, the most relevant conditions besides CURB-65 to predict the overall 30-day mortality in the adjusted analysis were chest X-ray abnormalities and leucopenia. For Ilg et al, a significant proportion of low-risk CAP also received critical care interventions such as vasopressors, inotropic support agents and assisted ventilation requiring ICU stay. ${ }^{24}$ Combined, this information suggests that CURB-65 demands further inspection and clinical decision tailoring when a low-risk category is assigned. In addition, patients with a higher length of stay should be closely followed to avoid readmissions.

The study results reflect participants with different clinical characteristics and income level based on healthcare plans. In this real-world study about CAP, no type of filter or selection was applied to include adult subjects. This should allow the results to be generalized to other middle-income countries. Moreover, given the retrospective nature of the study, and the fact that the information about pneumonia and other exposures were collected before the occurrence of death or readmission; therefore, it is unlikely that these outcomes had influenced the collection of information about pneumonia by the trained nurses. However, we detected some limitations to be discussed. Although our main result showed a reduction in the hospitalization of low-risk subjects linked to the implementation of the clinical pathway, these findings should be viewed with caution, since uncontrolled before and after studies are susceptible to biases related to temporality. Observed changes in admissions may have been due to variations in medical staff or patient comorbidity profiles such as smoking, diabetes, liver and renal impairment and heart failure, just to name a few. Also, doctors could be less likely to hospitalize a patient with low-risk CAP simple because they knew there was a clinical pathway being tested. A control group of hospitals where the clinical pathway had not been implemented would have permitted its effect to be best evaluated. Nevertheless, this investigation provides preliminary evidence for the effectiveness of such intervention.

Also, we controlled for many risk factors for death and readmission but we lack information about other important exposures already mentioned; thus, some residual confounding is likely to still be present changing our effect estimate. In addition, data were extracted from both medical records and administrative databases and users including the trained nurses might have different skills leading to potential variation in the information collected introducing a potential threat to internal validity. However, for the outcomes of interest - death and readmission - the information was based on ICD-10 codes captured from CDW even for outpatients, minimizing internal inconsistencies and misclassifications. The main reasons claimed for emergency physicians to admit patients with lowrisk CURB-65 were advanced age and the presence of comorbidities. However, this information was not systematically collected. To conclude, we could not detect that patients designated as low risk would be sent home as a result of higher levels of hospital occupancy leaving the attending physician with pressure to discharge.

\section{Conclusion}

The implementations of a clinical pathway encompassing both stakeholders and institutional support were successful in reducing the proportion of suspected low-risk CAP admissions with no apparent increase in 30-day outcomes. Nonetheless, controlled trials are necessary to confirm these results. Additional factors influence the clinical decision about site of care management and doctors should tailor when encountering a low leucocyte cell count and a chest radiography indicating the severity of the disease. The association of readmission risk in the low-risk population admitted to hospitals and a prolonged length of stay should be further explored.

\section{Abbreviations}

CAP, community-acquired pneumonia; CDW, Clinical Data Warehouse; COPD, chronic obstructive pulmonary disease; ER, emergency room; ICD-10, International Classification of Diseases; UHG, United Health Group.

\section{Data Sharing Statement}

The datasets generated and/or analyzed during the current study are not publicly available due to individual privacy that could be compromised but are available from the corresponding author on reasonable request.

\section{Ethics Approval and Consent to Participate}

This study was conducted according to the Declaration of Helsinki principles and was approved by the local research ethics committee of Pró-Cardíaco Hospital (Reference 
number 890). The institutional review board waived the requirement to obtain the informed consent based on the retrospective nature of the study, which involved minimal risks to subjects. Nevertheless, the investigators guarantee patient's confidentiality de-identifying the collect information of individual subjects and by anonymization of all data throughout the analysis.

\section{Acknowledgments}

We would like to thank the nurses responsible for collecting the data from medical charts and all the staff, clinicians and stakeholders involved in the implementation of the clinical pathway.

\section{Author Contributions}

All authors made substantial contributions to conception and design, acquisition of data, or analysis and interpretation of data; took part in drafting the article or revising it critically for important intellectual content; gave final approval of the version to be published; and agree to be accountable for all aspects of the work.

\section{Funding}

No funding was obtained for this study.

\section{Disclosure}

The authors declare that they have no competing interests to disclose related to the content of this manuscript.

\section{References}

1. Souza DK, Peixoto SV. Descriptive study on the evolution of hospitalization costs for ambulatory care sensitive conditions in Brazil, 2000-2013. Epidemiologia e Servicos De Saude. 2017;26 (2):285-294. doi:10.5123/S1679-49742017000200006

2. Mandell LA, Wunderink RG, Anzueto A, et al. Infectious Diseases Society of America/American Thoracic Society consensus guidelines on the management of community-acquired pneumonia in adults. Clin Infect Dis. 2007;44(Suppl 2):S27-72.

3. Mandell LA. Community-acquired pneumonia: an overview. Postgrad Med. 2015;127(6):607-615. doi:10.1080/00325481.2015.1074030

4. Fine MJ, Auble TE, Yealy DM, et al. A prediction rule to identify low-risk patients with community-acquired pneumonia. $N$ Engl J Med. 1997;336(4):243-250. doi:10.1056/NEJM199701233360402

5. Labarere J, Stone RA, Scott Obrosky D, et al. Factors associated with the hospitalization of low-risk patients with community-acquired pneumonia in a cluster-randomized trial. J Gen Intern Med. 2006;21 (7):745-752. doi:10.1111/j.1525-1497.2006.00510.x

6. File TM Jr., Marrie TJ. Burden of community-acquired pneumonia in North American adults. Postgrad Med. 2010;122(2):130-141. doi:10.3810/pgm.2010.03.2130
7. Blot SI, Rodriguez A, Sole-Violan J, et al. Effects of delayed oxygenation assessment on time to antibiotic delivery and mortality in patients with severe community-acquired pneumonia. Crit Care Med. 2007;35(11):2509-2514. doi:10.1097/01.CCM.0000287587.43 $801.9 \mathrm{C}$

8. Basi SK, Marrie TJ, Huang JQ, Majumdar SR. Patients admitted to hospital with suspected pneumonia and normal chest radiographs: epidemiology, microbiology, and outcomes. Am J Med. 2004;117 (5):305-311. doi:10.1016/j.amjmed.2004.03.029

9. Lim WS, van der Eerden MM, Laing R, et al. Defining community acquired pneumonia severity on presentation to hospital: an international derivation and validation study. Thorax. 2003;58(5):377-382. doi:10.1136/thorax.58.5.377

10. Haynes RB, Sackett DL, Gray JM, Cook DJ, Guyatt GH. Transferring evidence from research into practice: 1 . The role of clinical care research evidence in clinical decisions. ACP J Club. 1996;125(3):A14-16.

11. Guyatt GH, Rennie D. Users' guides to the medical literature. JAMA. 1993;270(17):2096-2097. doi:10.1001/jama.1993.03510170086037

12. Hersh WR, Crabtree MK, Hickam DH, et al. Factors associated with success in searching MEDLINE and applying evidence to answer clinical questions. J Am Med Inf Assoc. 2002;9(3):283-293. doi:10.1197/jamia.M0996

13. Guyatt G, Gutterman D, Baumann MH, et al. Grading strength of recommendations and quality of evidence in clinical guidelines: report from an american college of chest physicians task force. Chest. 2006;129(1):174-181. doi:10.1378/chest.129.1.174

14. Chalmers JD, Pletz MW, Aliberti S. Community-acquired pneumonia. Eur Respir Monographs. 2014;298.

15. RA LF C, Pereira-Silva JL. Diretrizes brasileiras para pneumonia adquirida na comunidade em adultos imunocompetentes. $J$ Bras Pneumol. 2009;35(6):574-601. doi:10.1590/S1806-3713200900060 0011

16. Grimshaw J, Eccles M, Tetroe J. Implementing clinical guidelines: current evidence and future implications. J Contin Educ Health Prof. 2004;24(Suppl 1):S31-37. doi:10.1002/chp.1340240506

17. Calcagno V. CM. glmulti: an R package for easy automated model selection with (Generalized) linear models. J Stat Softw. 2010;34 (12):29. doi:10.18637/jss.v034.i12

18. Fluss R, Faraggi D, Reiser B. Estimation of the Youden Index and its associated cutoff point. Biom J. 2005;47(4):458-472. doi:10.1002/ bimj. 200410135

19. Lee RW, Lindstrom ST. A teaching hospital's experience applying the Pneumonia Severity Index and antibiotic guidelines in the management of community-acquired pneumonia. Respirology. 2007;12 (5):754-758. doi:10.1111/j.1440-1843.2007.01121.x

20. Lim WS, Baudouin SV, George RC, et al. BTS guidelines for the management of community acquired pneumonia in adults: update 2009. Thorax. 2009;64(Suppl 3):iii1-55. doi:10.1136/thx.2009.121434

21. Cupurdija V, Lazic Z, Petrovic M, et al. Community-acquired pneumonia: economics of inpatient medical care vis-a-vis clinical severity. Jornal Brasileiro De Pneumologia. 2015;41(1):48-57. doi:10.1590/ S1806-37132015000100007

22. Blaeuer SR, Bally K, Tschudi P, Martina B, Zeller A. Acute cough illness in general practice - predictive value of clinical judgement and accuracy of requesting chest x-rays. Praxis. 2013;102 (21):1287-1292. doi:10.1024/1661-8157/a001437

23. van Vugt SF, Verheij TJ, de Jong PA, et al. Diagnosing pneumonia in patients with acute cough: clinical judgment compared to chest radiography. Eur Respir J. 2013;42(4):1076-1082. doi:10.1183/ 09031936.00111012

24. Ilg A, Moskowitz A, Konanki V, et al. Performance of the CURB-65 score in predicting critical care interventions in patients admitted with community-acquired pneumonia. Ann Emerg Med. 2018. 


\section{Publish your work in this journal}

The Open Access Emergency Medicine is an international, peerreviewed, open access journal publishing original research, reports, editorials, reviews and commentaries on all aspects of emergency
medicine. The manuscript management system is completely online and includes a very quick and fair peer-review system, which is all easy to use. Visit http://www.dovepress.com/testimonials.php to read real quotes from published authors.

Submit your manuscript here: https://www.dovepress.com/open-access-emergency-medicine-journal 